\title{
2019 closure, reviewers gratitude, and an invitation
}

\author{
Yoram Reich $^{1}$
}

Published online: 14 January 2020

○) Springer-Verlag London Ltd., part of Springer Nature 2020

Over the last year, the number of submissions to Research in Engineering Design dropped by about 20\%. This is explained by fewer submissions that are out of scope but also less good submissions. We published 27 papers, 15\% less than the year before and with $15 \%$ decrease in their total volume. We definitely need to attract more quality submissions and make sure we reduce the review time to do it. We intend to improve our processes and the overall service to authors and invite prospective authors to experience this firsthand.

None of this would have been possible without the extended community supporting the journal: the authors that trust the results of their research with the journal team, the area editors and the editorial board members, and the many dedicated reviewers.

I would like to extend my deep gratitude to the numerous reviewers who contributed their efforts to maintain and improve the high quality of the journal. The reviewers, outside the editorial board, who completed a review this year, are:

Achiche Sofiane
Allard Suzie
Allen Janet
Atlason Reynir
Baxter Weston
Ben-Arieh David
Bohm Matt
Borgianni Yuri
Brine Derek
Broniatowski David A.
Brown Christopher
Campbell Matthew
Campean Felician
Cavallucci Denis
Chen Wei
Chulvi Vicente
Coatanéa Eric
Cobb Corie
Culley Steve
de Neufville Richard
Dolah Rozzeta
Dori Dov
Dorst Kees
Earl Chris

Eifler Tobias
Eynard Benoit
Favi Claudio
Ferguson Scott
Fiorineschi Lorenzo
Franssen Maarten
Fritzsche Albrecht
Fu Katherine
Fukuda Shuichi
Garcia Agis Jose Jorge
Gericke Kilian
Goentzel Jarrod
Grogan Paul
Guenov Marin
Haftka Raphael
Hardt David E.
Hatchuel Armand
Hazelrigg George
Heimicke Jonas
Herder Pauline
Hoffenson Steven Hoffenson
Holt Raymond
Holtta-Otto Katja
Huang Chien-Yi

Huang Chen-I.
Ilevbare Imoh
Isaksson Ola
Jensen David
Jin Yan
Kannengiesser Udo
Katsikopoulos Konstantinos
Keshwani Sonal
Kimita Koji
Kipouros Timoleon
Koh Edwin
Kremer Gül
Kroll Ehud
Le Glatin Mario
Lenau Torben
Liu Yan
Lofthouse Vicky
Mackey Ryan
Matthiesen Sven
McAdams Daniel
McComb Chris
McDonald Libby
Mistree Farrokh
Mocko Greg

Yoram Reich

yoramr@tauex.tau.ac.il

1 Tel Aviv University, Tel Aviv, Israel 
Monplaisir Leslie

Moon Seung Ki

Moultrie James

Mourelatos Zissimos P.

Mulet Elena

Nagel Robert

Nakhla Michel

Oberoi Sharad

Ogura Masaki

Olewnik Andrew

Ostrosi Egon

Otto Kevin

Ottosson Stig

Proske Marina

Rai Bharatendra

Ramos Ana

Reich Yoram

Reiff-Marganiec Stephan
Ren Jingzheng

Rosen David

Ross Adam

Rotini Federico

Seepersad Carolyn

Shaked Avi

Sharma Sanjiv

Shen Weiming

Smulders Frido

Snider Chris

Sokol Julia

Sommer Svenja

Steinert Martin

Sudarsanam Nandan

Suh Eun Suk

Tacker Manfred

Tahera Khadija

Teissandier Denis
Thomas Maxime

Todeti Somasekhara Rao

TOH Christine

Ullah AMM Sharif

Ullman David

van Bossuyt Douglas

Venkataraman Srinivasan

Wang Ming-Feng

Wang Zheng

Wartzack Sandro

Whitcomb Clifford

Whitney Daniel

Whyte Jenny

Xiao Mi

Yang Maria

Yang Huayong

Yi-Min Deng

Zhao Fu

Publisher's Note Springer Nature remains neutral with regard to jurisdictional claims in published maps and institutional affiliations. 